\title{
Painful erythematous nodules in the left finger pads
}

Jeong Rae Yoo, Sang Taek Heo, Suhyun Oh, Seung-Jae Joo, and Ki Yung Boo

Department of Internal Medicine, Jeju National University School of Medicine, Jeju, Korea

Received: August 3, 2017

Revised : August 14, 2017

Accepted: January 15, 2018

\section{Correspondence to}

Sang Taek Heo, M.D.

Tel: $+82-64-754-8151$

Fax: +82-64-717-1131

E-mail: neosangtaek@naver.com
A 93-year-old woman presented to our emergency department with a 6-day history of left hand pain and swelling. On physical examination, a tender and erythematous nodule on the left finger pad (Fig. 1A) was identified and there was evidence of a pansystolic murmur,

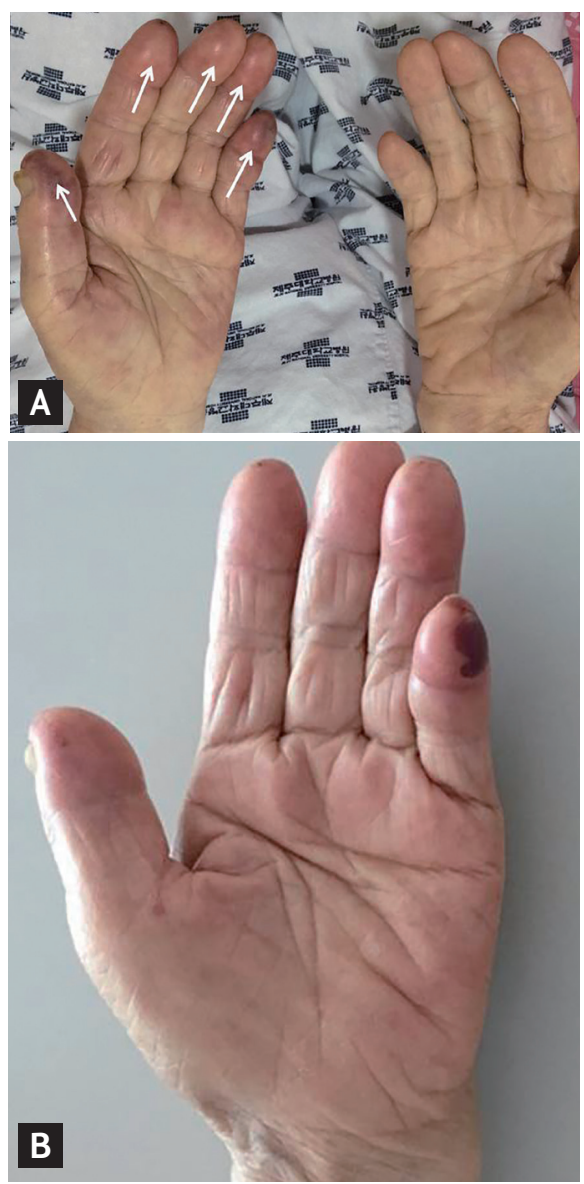

Figure 1. (A) Multiple osler's nodes overlying the left hand pads. (B) Swelling, tender, and erythematous nodules improving after 3 days of antibiotics treatment. Informed consent using pictures was obtained from patient and her family. which was more prominently heard at the apex of the heart. The patient had six teeth extracted 1 year before, followed by intermittent febrile episodes in addition to weight loss. An upper extremity angiography and computed tomography revealed no definite arterial stenosis or occlusion of any artery in the upper extremities. Initially, she had no fever, and laboratory findings revealed a total white blood cell count of $8,900 \times 10^{3} / \mu \mathrm{L}$ (normal range 6,000 to 10,000$)$, a C-reactive protein level of $1.35 \mathrm{mg} / \mathrm{dL}$ (normal range, 0.00 to 0.30 ), and an erythrocyte sedimentation rate of $62 \mathrm{~mm} / \mathrm{hr}$ (normal range, o to 20 ). Serial blood cultures revealed no growth of any pathogens for 2 weeks. Transthoracic echocardiography revealed the presence of vegetation $\left(0.66 \times 0.20 \mathrm{~cm}^{2}\right)$ on the atrial side of the anterior mitral valve leaflet and thickening of the aortic valve with a small vegetation $(0.45 \times 0.35$ $\mathrm{cm}^{2}$ ) (Fig. 2). Hence, the diagnosis of infective endocarditis was made on the basis of modified Duke's criteria. The patient was subsequently started on ceftriaxone $2 \mathrm{~g} /$ day and gentamicin 1 $\mathrm{mg} / \mathrm{kg}$ and the lesion showed improvement with this antibiotic regimen (Fig. ${ }_{1 B}$ ). However, the patient was hopeless discharged for advanced aging.

Olser's nodes are tender, raised nodules on the pads of the fingers or toes, whereas Janeway lesions are usually non-tender and found on the palms or soles. Osler's nodes are the result of micro septic emboli from valvular 

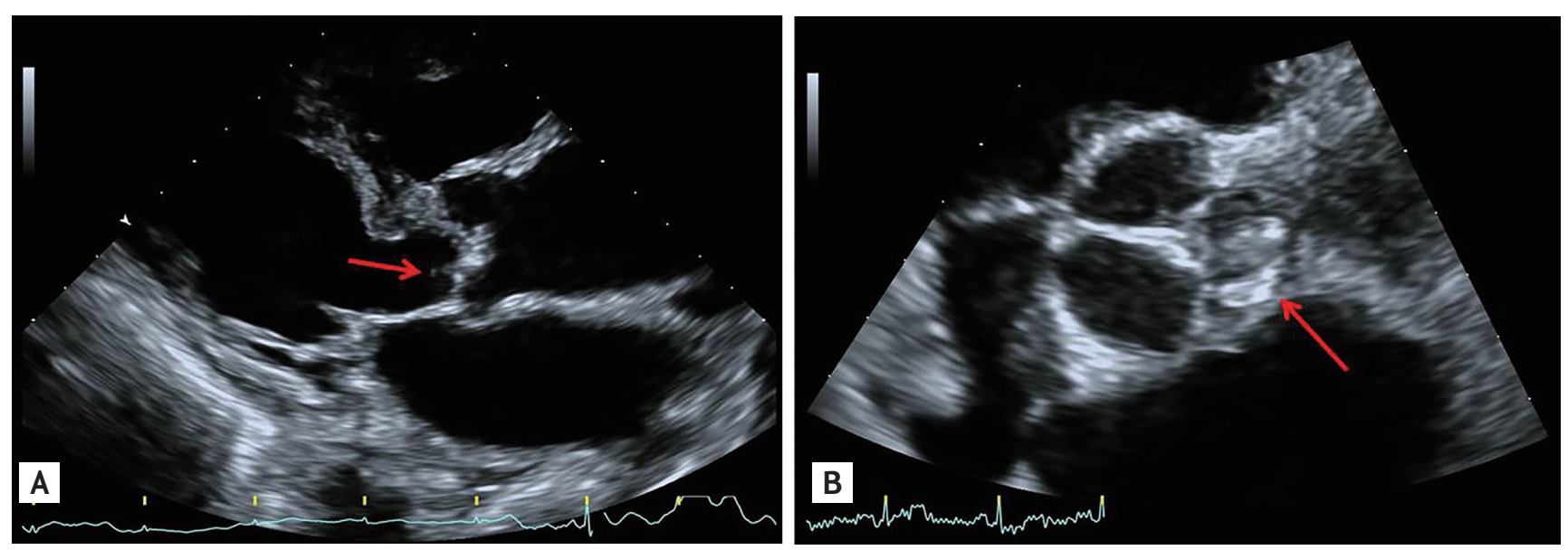

Figure 2. (A) Red arrow, avegetation on the aortic valve in the parasternal long axis view. (B) Red arrow, a vegetation on aortic valve in the parasternal short axis five chamber view.

vegetations that cause dermal microabscesses to form in patients with infectious endocarditis. Conversely, subacute bacterial endocarditis tends to produce lesions with vasculitic changes which are characteristic of Janeway lesions.

\section{Conflict of interest}

No potential conflict of interest relevant to this article was reported. 